\title{
The Research on Synthesis of Polyacrylamide with Photo-Initiation
}

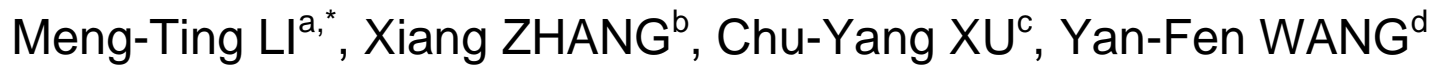

School of Materials Science and Engineering, Anhui University of Science \& Technology, Huainan 232001, Anhui Province, P.R. China

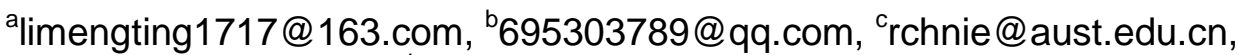

'wangyanfenyu@163.com

${ }^{*}$ Corresponding author

Keywords: CPAM, Photo-initiation, Solid Content, Intrinsic Viscosity.

\begin{abstract}
This paper has studied the influence of different initiation of flocculants synthesis, the effect of flocculant products based on solid content and characteristic viscosity indicators to the performance on different types of polyacrylamide products, and then chose the best one as the research object. The optimal dosage and other influencing factors were researched just for getting the best solution for synthesizing.
\end{abstract}

\section{Introduction}

With the continuous improvement of social productivity and urbanization accelerating, there is a sharp rise in demand of water resources of people in the production and life. However serious water pollution has become one of the most serious ecological crisis in the world which is facing. In response to increasingly serious shortage of global water resource, and to slow the more serious trend of water pollution, all kinds of methods were researched at home and abroad [1]. The flocculation settlement in the wastewater treatment has its obvious effects of cost reduction and convenience, is getting more attention from people. At present, the research of polyacrylamide production technology and polymerization methods are getting more attention [2]. Therefore, how to increase the molecular weight of polyacrylamide for improving its dissolution performance and improving the flocculation effect need a further study for exploration of important project.

\section{Experimental}

In this part, the experimenter respectively took three ways of light trigger, chemical initiation and thermal initiation for synthesis of cationic polyacrylamide as the research object. Monomer is composed of acrylamide and DMC, and the ratio of $n(A M): n(D M C)=1: 0.2$. The preliminary design monomer mass fraction is $32 \%, 35 \%, 38 \%$. The photo-initiation refers to tracing photosensitive initiator under the sunlight or ultraviolet-light. Thermal-initiation is pointed to by adding potassium persulfate as the initiator into monomer mixture, controlling the temperature in the oven, thus providing the temperature needed for the reaction conditions of polymerization. Chemical- initiation refers to adding potassium persulfate and sodium thiosulfate as the initiator to the monomer mixture. Sodium thiosulfate can help the former polymerization under the condition of without high temperature, it can also be referred to as the additives-initiator. These experiments were made in the same laboratory under alike environment, with sufficient reaction time, and finally by measuring each group of viscosity to characterize the advantages and disadvantages. The experimental results were shown in the figure.1.

The steps of synthesis for cationic polyacrylamide are as follows: First, white solid powder polyacrylamide was added to the reaction flask by using a spoon. Then use glue dropper to suck up DMC, sticky transparent liquid drops to the white powder. Next, deionized-water was added to the monomer mixture zone, while the exploratory normal ratio of monomer mass fraction was 35\%, stir well. After the monomers completely were dissolved, photosensitive initiator amount was very small, in accordance with the proportion of $0.01 \%, 0.03 \%, 0.05 \%, 0.07 \%, 0.09 \%, 0.11 \%$ added to the mixed monomer solution in and stir evenly. The solution was filled with nitrogen for four 
minutes, removed the oxygen, for preventing product oxidation turning to yellow and hard in the process of polymerization. The oxygen displacement solution was wrapped with preservative film,solution has been successfully polymerized, when the reaction solution shaped into jelly under the sunlight or ultraviolet light exposure for 0.5-1h [3]. Performance test of the product can be carried on about one day later. The synthetic process of APAM and ACPAM was roughly similar with above sample. Due to the different monomers, anionic polyacrylamide was synthesized from acrylamide and acrylic acid, their ratio was 3:1, while amphoteric acrylamide was compounded of DMC, acrylic acid and sodium hydroxide, their ratio was 1:0.18:0.65. Please look at the experimental results which were shown in figure 2.

\section{Results and Discussion}

Like the pictured 1, the characteristic viscosity of flocculating agents by three ways have very big differences. The characteristic viscosity of flocculant by photo-initiation was significantly greater than chemical-initiation and thermal-initiation, namely light polymerization were more likely to get high molecular weight polyacrylamide flocculant.

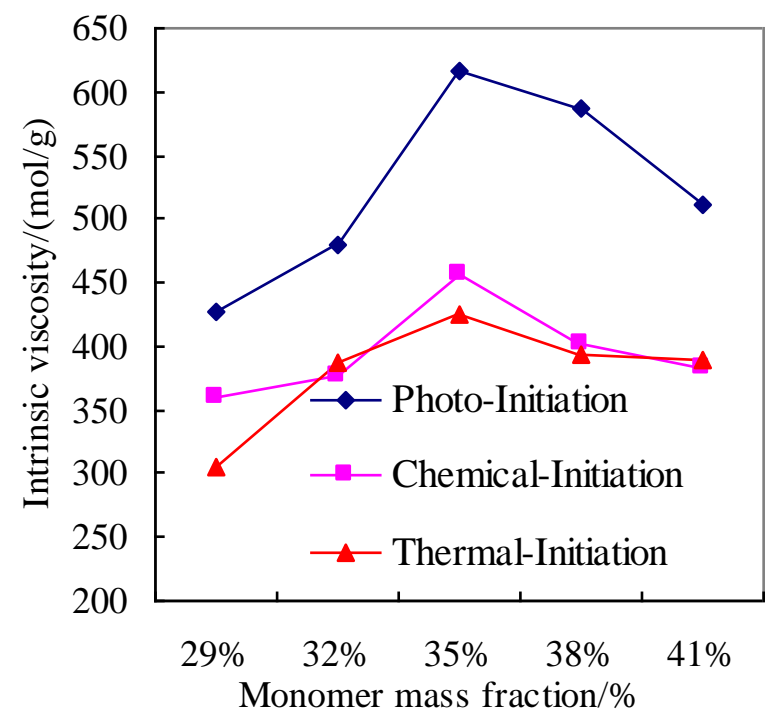

Fig.1 The effect of flocculant characteristic viscosity on different trigger methods

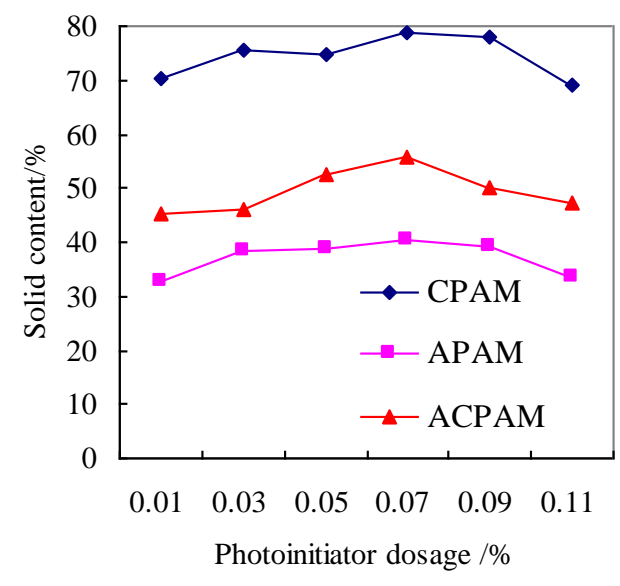

(a) and on intrinsic viscosity

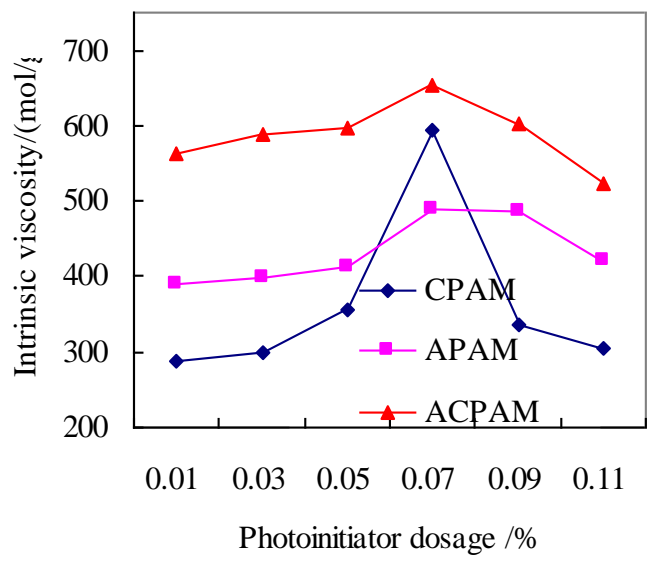

(b) for different kinds of flocculant

Fig.2 The effects of different photoinitiator dosage on solid content

Under the condition of light trigger for polymerization polymerization to produce polyacrylamide, 
two points were seen in Fig. 2. On one hand, when the initiator content is about in the range of $0.07 \% \sim 0.09 \%$, the solid content and characteristic viscosity of the product all reached the peak value, so it was the best process parameters of polymerization. On the other hand, the three kinds of flocculants monomer were different, while the initiator content, monomer mass fraction and other conditions were in the same circumstances, the solid content and characteristic viscosity of the product were different. By contrast, the solid content and characteristic viscosity of cationic polyacrylamide were the maximum value, so the cationic eventually was selected as the research object [4].

\section{Conclusions}

After Comprehensive comparison, a novel amphoteric chemically bonded CPAM flocculant was designed and successfully prepared by the photo-initiated polymerized route. The solid content and characteristic viscosity of products reached the peak when the initiator content is about $0.08 \%$. The molecular weight of flocculant realized the maximum when the monomer mass fraction is $35 \%$. This paper can well promote the development of high-performance flocculants.

\section{References}

[1] Ying Hui Ngo, Dan Li, George P. Simon, Gil Garnier. Effect of cationic polyacrylamides on the aggregation and SERS performance of gold nanoparticles-treated paper[J]. Journal of Colloid and Interface Science.,2013,392: 237-246.

[2] Zhong Lian Yang, Bao Yu Gao, Chun Xiao Li, Qin Yan Yue, Bin Liu. Synthesis and characterization of hydrophobically associating cationic polyacrylamide[J].Chemical Engineering Journal, 2010,161: 27-33.

[3] Kamilla da Mata, Marcela Zanetti Corazza, Fernanda Midori de Oliveira, Ana Lúcia de Toffoli, César Ricardo Teixeira Tarley, Altair Benedito Moreira. Synthesis and characterization of cross-linked molecularly imprinted polyacrylamide for the extraction/preconcentration of glyphosate and aminomethylphosphonic acid from water samples[J]. Reactive and Functional Polymers, 2014,83:76-83.

[4] M. Ejtemaei, A. Tavakoli, N. Charchi, B. Bayati, A.A. Babaluo, Y. Bayat. Synthesis of sulfated zirconia nanopowders via polyacrylamide gel method[J]. Advanced Powder Technology, 2014, 58:251-258. 